\title{
Escuchando a las víctimas del conflicto armado colombiano: la experiencia de un dispositivo de atención psicosocial
}

DOI: http://dx.doi.org/10.18046/recs.i25.2696

\begin{abstract}
Listening to the Victims of the Colombian Armed Conflict: The Experience of a Mechanism for Psychosocial Attention

Escutando as vitimas do conflito armado colombiano: a experiência de um dispositivo de atenção psicossocial
\end{abstract}

\author{
XIMENA CASTRO-SARDI ${ }^{* *}$ \\ MARGARITA MUNÉVAR-ROJAS ${ }^{* * *}$
}

Universidad Icesi (Cali, Colombia)

\footnotetext{
* Este artículo es resultado del proyecto de investigación «Clínica del trauma con víctimas de la violencia: de la victimología al psicoanálisis» (2015-2018) financiado por la Universidad Icesi. Además de las autoras de este artículo, han participado en este proyecto los estudiantes Juan David Paz y María Claudia Crespo, y la asistente de investigación Juliana Olano. Artículo de investigación recibido el 15-01-2018 y aceptado el o6-04-2018.

** Profesora del Departamento de Estudios Psicológicos y Directora del Consultorio de Atención Psicosocial (CAPsi), Universidad Icesi. Candidata a doctora en Psicoanálisis, Universidad París 8. Línea de investigación: psicoanálisis aplicado a la intervención social y a la salud mental. Correo electrónico: xcastro@icesi.edu.co ORCID: https://orcid. org/oooo-0oo2-0740-9293

*** Profesora del Departamento de Estudios Psicológicos y Psicóloga del Consultorio de Atención Psicosocial (CAPsi), Universidad Icesi. Magister en Intervención Psicosocial, Universidad Icesi. Correo electrónico: mmmunevar@icesi.edu. co ORCID: https://orcid.org/oooo-ooo3-0418-3070
} 


\section{Cómo citar}

MunÉvar-Rojas, M. y CASTRO-SARDI, X. (2018). Escuchando a las víctimas del conflicto armado colombiano: la experiencia de un dispositivo de atención psicosocial. Revista CS, 25, 81-109. http://dx.doi.org/10.18046/recs.i25.2696 
Resumen

Abstract

Resumo

El reconocimiento por parte del Estado colombiano de la necesidad de establecer mecanismos de reparación integral de las víctimas del conflicto armado ha desembocado en propuestas de rehabilitación psicosocial cuyo fin es mitigar las afectaciones mentales causadas por los hechos violentos. Este artículo presenta la experiencia de un dispositivo de atención psicosocial con miras a contribuir a la reflexión sobre la necesidad y efectividad de las intervenciones propuestas. Se presentan tres casos de mujeres víctimas atendidas y se analizan teniendo en cuenta tres categorías: las implicaciones subjetivas del reconocimiento social de "víctima», la elaboración psíquica del acontecimiento traumático y los alcances del proceso de intervención psicoterapéutica. Se concluye acerca de la importancia de sostener una escucha atenta a las coordenadas singulares de cada sujeto-víctima, evitando el supuesto de que las afectaciones del hecho victimizante puedan generalizarse, y de la necesidad de fortalecer el trabajo en red entre instituciones y dispositivos asistenciales.

\section{PALABRAS CLAVE:}

conflicto armado colombiano, víctimas, rehabilitación psicosocial, psicoanálisis aplicado, subjetividad

The recognition on the part of the Colombian state of the necessity of establishing mechanisms for the full compensation of the victims of the armed conflict has resulted in proposals of psychosocial rehabilitation aimed at mitigating the lasting mental effects caused by violent events. This article presents the experience of one instrument of psychosocial attention with the aim of contributing to the reflection about the necessity and the effectiveness of the proposed interventions. It presents the cases of three female victims and analyses them, taking three categories into account: the subjective implications of social recognition of the «victim», the psychological development of the traumatic event, and the scope of the process of psychotherapeutic intervention. It arrives at conclusions 
about the importance of maintaining attentive listening to the individual coordinates of each subject-victim; avoiding the assumption that the long-term effects of victimizing events can be generalized; and of the necessity of strengthening the networking between institutions and healthcare services.

\section{KEYWORDS:}

Colombian armed conflict, Victims, Psychosocial rehabilitation, Applied psychoanalysis, Subjectivity

O reconhecimento por parte do estado colombiano da necessidade de estabelecer mecanismos de reparação integral das vítimas do conflito armado desembocou em propostas de reabilitação psicossocial cujo fim é mitigar as afetações mentais causadas pelos atos violentos. Este artigo apresenta a experiência de um dispositivo de atenção psicossocial com olhadas para contribuir com a reflexão sobre a necessidade e a efetividade das intervenções propostas. Apresentam-se três casos de mulheres vítimas atendidas e analisam-se tendo em levando em consideração três categorias: as implicações subjetivas do reconhecimento social de «vítima», a elaboração psíquica do acontecimento traumático e dos alcances do processo de intervenção psicoterapêutica. Se conclui sobre a importância de suster uma escuta atenta às coordenadas singulares de cada sujeito-vítima, evitando o suposto de que as afetações do ato de vítima possam generalizar-se, e da necessidade de fortalecer o trabalho em rede entre instituições de dispositivos assistenciais.

\section{PALAVRAS CHAVE:}

conflito armado colombiano, vítimas, reabilitação psicossocial, psicanálise aplicada, subjetividade 


\title{
Introducción
}

\begin{abstract}
Nuestras almas no encuentran sosiego porque no hemos podido hacer un auténtico duelo a los angelitos que perdimos, a las mujeres embarazadas, madres, padres, hermanos... que fueron arrancados de nuestro territorio.
\end{abstract}

Con estas palabras se manifestó el Comité de Víctimas el 6 de diciembre de 2015 en Bellavista viejo (Bojayá, Chocó), en el acto de perdón organizado entre los sobrevivientes de la masacre de Bojayá y una delegación de las FARC. La masacre de Bojayá es solo uno de muchos ejemplos sobre las instancias en las que el Estado y demás instituciones les han fallado a las víctimas del conflicto armado en Colombia, especialmente en términos de salud mental. Su sufrimiento, quizás pasado por alto en ciertos casos, se manifiesta en dolores físicos y sufrimiento agudo, como si no hubiera pasado un día desde su tragedia y desplazamiento. Lo anterior no solo da cuenta de la insuficiencia de los mecanismos de reparación que se han puesto en marcha, sino que constituye un llamado a las instituciones y los agentes interventores para que se diseñen y propongan formas efectivas de atender y tratar el sufrimiento psíquico y social consecuencia del conflicto armado colombiano.

Desde el marco jurídico colombiano, la Ley 1448 de 2011, conocida comúnmente como la Ley de Víctimas, que reconoce a las víctimas del conflicto armado y establece las medidas de reparación de los daños causados, ha sido uno de los instrumentos legales propuestos por el Estado en el camino hacia la construcción de la paz. Una de las modalidades que establece dicha ley para reparar a esta población vulnerada es la rehabilitación, definida como un «conjunto de estrategias, planes, programas y acciones de carácter jurídico, médico, psicológico y social, dirigidos al restablecimiento de las condiciones físicas y psicosociales de las víctimas» (República de Colombia, 2011: 63). Asimismo, se establece que el acompañamiento psicosocial debe ser transversal al proceso de reparación, para lo cual se establecen los lineamientos del Programa de Atención Psicosocial y Salud Integral a Víctimas, PAPSIVI (Ministerio de Salud y Protección Social, 2017).

Uno de los grandes avances de esta ley es la ampliación de los criterios de reconocimiento de las víctimas: se identifican como víctimas a todas aquellas personas que hayan vivido un evento del conflicto armado que haya vulnerado sus derechos humanos o hayan sufrido la pérdida de un familiar; lo que implica efectos por vía de la reparación administrativa (Bolaños, 2011; Gustin y Palau, 2013). Este es un avance respecto a la anterior Ley 975 de Justicia y Paz, dado que uno de los riesgos que se corría con esa ley era que las víctimas se convirtieran en objetos de indemnización del Estado y no en sujetos de derecho (Salgado, Nobles y Ruiz, 2011). Sin embargo, la 
ley no se encuentra libre de críticas. Las víctimas no tuvieron participación durante su formulación, acto que las ubica en una posición pasiva, y como simples beneficiarias del saber de los expertos (Bolaños, 2011; Gustin y Palau, 2013). Las víctimas, además, son consideradas de forma individual, desconociendo que esta forma de sufrimiento ha sido producida socialmente (Aguilera, 2013), dando lugar a una privatización de los daños vividos por estas comunidades (Centro de Memoria Histórica, 2013).

Adicional a lo anterior, a partir del informe de seguimiento realizado por Quintero, Recalde, Gómez y Flechas (2015) respecto a la implementación de políticas públicas en materia de salud mental en niños y adolescentes desplazados, se identificaron algunas limitaciones relacionadas con la atención psicosocial que se está prestando a las víctimas, a través de la aplicación de la ley mencionada. Una de ellas, la cual se encuentra estrechamente relacionada con la ausencia de coordinación entre las entidades territoriales de salud y el nivel central del Estado, es «la separación del componente de salud mental de los programas de rehabilitación psicosocial para población desplazada» (Quintero, Recalde, Gómez y Flechas, 2015: 2). Según este informe, el problema yace en que las políticas públicas separan el componente de salud mental de los programas de rehabilitación psicosocial, de manera que la población desplazada se enfrenta a un paradigma de atención enfocado en el trastorno y la atención psiquiátrica de manera exclusiva (Quintero et al., 2015). Según esto, las víctimas obtienen solo una atención parcial, desligada de las necesidades integrales de sus vidas y con fuertes tendencias a la medicalización. Se podría decir incluso que, a pesar de las múltiples rutas de atención que existen, pocas brindan la atención psicosocial tal y como se ha definido en el marco legal.

\section{Abordajes de las afectaciones psicológicas en el contexto del conflicto armado colombiano}

Desde principios de este siglo y a partir de los hechos atroces de violencia en el marco del conflicto armado en nuestro país, se comenzó a establecer la necesidad de identificar, caracterizar y tratar las afectaciones psicológicas como consecuencia de los acontecimientos violentos, tales como masacres, secuestros, desapariciones y desplazamientos forzados.

Con el fin de caracterizar el estado de la salud mental de las víctimas de la masacre ocurrida el 2 de mayo de 2002 en Bojayá, Londoño et al. (2005) realizaron un estudio cuantitativo para medir los índices de estrés postraumático, aplicando en una muestra de 40 adultos la escala MINI (Mini Internacional Neuropsychiatric Interview), que evalúa los principales trastornos psiquiátricos del eje I del DSM-IV y el CIE-1O. 
Según este estudio, el 37\% de los participantes presentó estrés postraumático, y todos ellos habían retornado a Bojayá. Los trastornos que más se relacionaron al estrés postrauma fueron la fobia social, trastornos por angustia y agorafobia.

Por su parte, Botero (2004) hace una investigación cuasi-experimental que busca establecer la efectividad de las técnicas grupales cognitivo-conductuales de exposición prolongada e inoculación de estrés en 42 miembros de las fuerzas armadas en procesos de rehabilitación con diagnóstico de estrés postraumático. Para el desarrollo de la investigación se realizó una serie de tests, como el inventario de Depresión de Beck y la escala de Diagnóstico del Desorden por Estrés Postraumático de Foa. En el análisis de los resultados se utilizaron pruebas estadísticas de pre y post, a partir de las cuales se pudo establecer una reducción significativa de los síntomas y signos del estrés postrauma al finalizar el proceso de intervención.

Puede decirse que en estos abordajes prima el modelo de la psiquiatría biológica, en la cual «el síntoma, hecho signo, define la enfermedad. [...] [en este sentido] la biografía del sujeto es, como mucho, secundaria» (Desviat, 2015:12). Puede decirse que en estas investigaciones la historia, tanto subjetiva como comunitaria, resulta poco relevante dentro del curso de la intervención, adquiriendo mayor importancia los signos de enfermedad que permitan objetivar el sufrimiento psíquico para dotarlo de cualidad científica-positivista. Una de las consecuencias de esta objetivación es la reducción del trauma a un evento que puede ser definido a priori y generalizado a todos los sujetos, desconociendo la dimensión subjetiva y singular de la vivencia traumática (Gómez, 2004).

Desde otra perspectiva, el Programa de Atención Psicosocial y Salud Integral a Víctimas (PAPSIVI) focaliza su atención en el daño y las afectaciones psicosociales derivadas del mismo, y a partir de ello opera con un modelo de diagnóstico del que derivan la realización de una serie de intervenciones inscritas en el paradigma cognitivo-conductual (Ministerio de Salud y Protección Social, 2017).

Adicionalmente, a partir de la revisión bibliográfica se encuentran reflexiones sobre intervenciones desde la perspectiva psicosocial (Aguilera, 2013; García, 2012; Latorre, 2010; Wilches, 2010), en las cuales se conciben las víctimas como sujetos participantes y activos, capaces de gestionar sus propios cambios sociales; y también se le da lugar al experto en el campo de la acción social, entendiéndolo como un facilitador, un catalizador de esos cambios gestionados por la propia comunidad víctima del conflicto armado. Desde esta perspectiva, además se plantea, como herramienta de intervención, la utilización de elementos simbólicos que permitan al sujeto nombrar al sufrimiento, acotarlo en palabras. Un ejemplo de ello puede encontrarse en la promulgación de la memoria colectiva como instrumento de resistencia ante la historia actual del conflicto armado, buscando lograr, a partir de ello, 
el reconocimiento de las víctimas como sujetos de derecho, en pro de reconstruir sus vidas y lograr una reinserción social.

Por su parte, Rebolledo y Rondón (2010) desarrollan un concepto de daño individual y colectivo, así como modalidades de evaluación y de intervención para una reparación integral. Las autoras advierten acerca de las intervenciones que solo tratan lo individual desde una perspectiva médica del estrés postraumático, que se centran en la narración de los eventos dolorosos y pueden traer como consecuencia la alienación de las personas. Por este motivo, acuden a la definición de daño como proceso y no como hecho puntual, que llega a vulnerar la dignidad del sujeto y no se restringe a una afectación individual, sino que perturba su sistema de relaciones $\mathrm{y}$, en esa misma medida, al conjunto comunitario al que pertenece.

Por otra parte, Gutiérrez-Peláez (2017) reflexiona acerca de la importancia de reconocer y dar lugar, dentro del marco de las intervenciones psicosociales, a los recursos, herramientas y construcciones propias con los que cuentan las comunidades para afrontar sus problemáticas. Al respecto, el autor plantea que los profesionales de la salud mental tienden a caer en una trampa llamada la falsa unificación de las muestras, la cual los lleva a considerar homogéneos a grupos que en la práctica pueden resultar radicalmente diferentes, a pesar de pertenecer a un mismo espacio y a una misma temporalidad. En este sentido, este autor sostiene que las intervenciones psicológicas y psicosociales que se implementen en diferentes comunidades y regiones:

Tienen que tener en cuenta las soluciones que cada una de esas comunidades ha construido para responder a sus problemáticas. No hay un meta-discurso o un plan de intervención que pueda prescindir del saber de cada comunidad y de cada sujeto (Gutiérrez-Peláez, 2017:3).

Considerando lo anterior, y teniendo como telón de fondo la diversidad de modos y enfoques empleados con el fin de hacer efectiva la llamada atención psicosocial, consideramos pertinente presentar la experiencia de un dispositivo asistencial creado por el Programa de Psicología de la Universidad Icesi de Cali: el Centro de Atención Psicosocial (CAPsi), que ofrece un servicio de acompañamiento centrado en la escucha psicológica cualificada a poblaciones vulnerables, entre las que se encuentran las víctimas del conflicto armado y de otras formas de violencia. El presente artículo tiene como propósito analizar la experiencia de este dispositivo a través de tres estudios de caso de víctimas atendidas, con miras a contribuir a la reflexión sobre las estrategias necesarias para hacer efectivas la reparación y rehabilitación psicosocial.

Ahora bien, con el fin de contextualizar la postura epistemológica desde la cual se sitúa la propuesta de intervención del dispositivo CAPsi, resulta pertinente iniciar 
por establecer algunas precisiones teóricas acerca de tres conceptos que devienen esenciales para definir el funcionamiento de dicho dispositivo, a saber: víctima, trauma y rectificación subjetiva.

\section{Sobre la condición de víctima y el trauma}

Para definir este concepto, situándolo particularmente en el marco de la violencia, resulta pertinente retomar lo planteado por Arias (2012), quien a través del desarrollo de una exposición crítica acerca de las concepciones históricas y contemporáneas del concepto de víctima, presenta la descripción de dos posturas que se han constituido como tradicionales sobre el tema, a saber, la postura sacrificial de la Iglesia católica y la postura asumida por el derecho penal. De acuerdo con lo planteado por este autor, dentro del campo del derecho penal, se reduce su definición a la de un sujeto pasivo del delito tipificado, avivando con ello su contenido sacrificial, de modo que se encubren bajo esta concepción contenidos de exclusión, marginalidad, menosprecio, auto-conmiseración y bajo potencial de protesta en referencia al sujeto considerado como víctima.

Las ideas de posición sacrificial de las víctimas solo consideran de ellas su cuerpo sufriente; sin embargo, para Arias (2012) solo es a partir de las víctimas que podemos conocer el vínculo entre los derechos humanos y la dignidad. En concordancia, para definir el concepto de víctima resulta necesario trascender el cuerpo sufriente y postrado de la víctima, a un cuerpo-idea que no se agota en el sentimiento moral de compasión, así la víctima será testigo de algo más que de sí misma: que sea un cuerpo vehículo del empoderamiento, de un propósito de resistencia y emancipación (Arias, 2012).

En concordancia con lo anterior, para efectos del presente artículo, se entenderá el concepto de víctima: primero, como un cuerpo que ha devenido sufriente como resultado de cualquier hecho violento que haya generado directa o indirectamente la vulneración de su dignidad humana; teniendo en cuenta que no solo son vulnerantes las acciones violentas que actúan directamente sobre el cuerpo, sino también todas aquellas que actúan sobre el entorno de relaciones significativas que rodean dicho cuerpo, sobre el contexto social de la persona; segundo, como un sujeto cuyas potencialidades trascienden el carácter de cuerpo sufriente y que posee la capacidad de afrontar los hechos traumáticos que han marcado su historia de vida, desde una moción emancipatoria y de empoderamiento que le permita «construir una salida al trauma que no lo deje atrapado en la identificación a la pasión sacrificial de la víctima» (Giraldo, 2016: 1). 
Con el propósito de realizar un abordaje comprensivo del trauma, el desarrollo conceptual del presente artículo se sitúa desde la perspectiva psicoanalítica, dado que esta permite tomar distancia de posturas individualistas y posturas que resaltan la pasividad sacrificial de las víctimas; permitiendo situar la responsabilidad de los sujetos frente a su sufrimiento (Giraldo, 2016).

En este sentido, Arciniegas (2012) plantea que «el trauma no es tanto un acontecimiento en sí, sino la vivencia del sujeto respecto a un acontecimiento» (44). Se trata de una vivencia emocional particular a cada sujeto (o grupo social) que introduce una discontinuidad en la vida del mismo (existe un «antes del trauma» y un «después del trauma»); una que resulta difícil de resolver porque representa algo inesperado y en ocasiones ininteligible para el sujeto desde sus vivencias y experiencias anteriores (Arciniegas, 2012). Por consiguiente, el trauma se basa en la imposibilidad de hilar esta vivencia con el resto de la experiencia vital, dificultando el establecimiento de un antes y un después, entorpeciendo en consecuencia la significación de la vivencia por parte de la persona (Efraime, 2017).

\section{La escucha como propuesta de intervención y la rectificación subjetiva}

Desde las perspectivas teóricas sobre la subjetividad, como la psicoanalítica expuesta por Miller (2004), se plantea que la escucha como herramienta de intervención cumple un rol fundamental en la sociedad, ya que a partir de esta se provee al sujeto un espacio para nombrar su sufrimiento de forma singular, permitiéndole encontrar una salida simbólica al mismo como alternativa a las manifestaciones sintomáticas de la contemporaneidad, como los pasajes al acto violento, los consumos adictivos o los trastornos alimenticios.

Desde esta aproximación, la atención psicológica prioriza una escucha desprejuiciada, donde el terapeuta se ubica frente al paciente desde una posición de no-saber (Miller, 2004) que pueda favorecer en el sujeto sufriente la posibilidad de erigir sus propias respuestas, su propia invención, y que le permita reconstruir su lazo social, convirtiéndose en agente activo de dicho proceso. Se parte del principio de la singularidad, entendiendo que, aunque compartan un espacio sociocultural común, no todos los sujetos son iguales, de modo que cada uno en su construcción subjetiva puede encontrar por sí mismo recursos que le ayuden a afrontar las experiencias traumáticas por las cuales ha atravesado, y tramitar de algún modo el padecimiento derivado. Asimismo, en un espacio de escucha se pueden identificar y alojar los otros modos de reparación que no hayan sido contemplados por la ley, reconociendo que 
no hay mecanismo institucional que pueda prever y atender a los modos particulares de elaborar y significar la experiencia vivida del horror (Bravo, 2016).

En concordancia con lo anterior, resulta relevante situar el concepto de rectificación subjetiva como un elemento necesario dentro del dispositivo de intervención a través de la escucha que se plantea. Tomando como referencia las concepciones iniciales postuladas por Lacan (citado por Miller, 2004) acerca de este concepto, podría señalarse que la rectificación subjetiva sucede cuando, durante un análisis, el sujeto aprende o logra situar también su responsabilidad esencial en lo que ocurre; sin embargo, puede decirse que más allá de situar la responsabilidad o la implicación del sujeto en lo que le acontece, la rectificación subjetiva consiste en que el sujeto logre introducirse en una primera localización respecto a su posición en lo real (Miller, 2004). Lo anterior implica que el sujeto que acude a un dispositivo de escucha requiere poder entrar en contacto a través de la palabra con esa disrupción en su ser; poder situar frente a un Otro esa falta en el ser, ese imposible que resulta indialectizable e inviable de hilar con el resto de su experiencia vital, y a partir de ello tomar una posición de agenciamiento y empoderamiento respecto al curso del afrontamiento de su malestar y su trauma psíquico, en el marco de la relación transferencial.

\section{El Consultorio de Atención Psicosocial (CAPsi)}

Desde la apertura del Consultorio de Atención Psicosocial (CAPsi) en el año 2014, se ha atendido un total de 700 consultantes, los cuales provienen de estratos socioeconómicos 1, 2 y 3, de las zonas vulnerables de la ciudad de Cali, como el Distrito de Aguablanca, los barrios ubicados en las laderas occidentales, entre otros (Castro, 2016). ${ }^{1}$ La propuesta de intervención del consultorio se enmarca en los lineamientos de formación clínica del programa de psicología de la Universidad Icesi, los cuales se fundamentan en una concepción psicosocial de la subjetividad; una aproximación que cuestiona la dicotomía individuo-sociedad y entiende el malestar del sujeto en relación con los contextos y significados sociales que lo constituyen. De este modo, la perspectiva del CAPsi se sitúa en una mirada psicosocial que reconoce que el objeto de las acciones en salud mental no es, de un modo exclusivo, el individuo o los conjuntos sociales, sino las relaciones que permiten pensar conjuntamente al individuo y su comunidad. El desarrollo de esta forma de entender la salud mental se sostiene en una ética que considera:

1. El capítulo de Castro (2016) presenta en mayor detalle la caracterización sociodemográfica y de motivos de consulta de la población atendida en el CAPsi. 
[...] al sujeto del sufrimiento mental, su historia, su sensibilidad, su experiencia y su memoria, la dimensión conflictiva de toda existencia humana y propone al sujeto una comprensión conjunta del malestar psíquico, esto es, su participación en el proceso de atención. (Galende, 2008: 26).

A partir de lo anterior, surge la construcción de un dispositivo de intervención clínico-comunitario en el que se reconoce la utilidad que tiene la escucha en el fortalecimiento del tejido social; un espacio de escucha psicosocial que permite que los consultantes puedan hablar de su sufrimiento con el fin de construir salidas simbólicas frente a su malestar, apoyándose en sus recursos subjetivos y en sus redes comunitarias, y restituyendo así su lugar activo en el restablecimiento de sus derechos y del lazo social (Castro y Munévar, 2016).

\section{Método}

Esta investigación está enmarcada en una epistemología cualitativa y se basa en una metodología de estudios de caso, en cuanto se constituyen como «una fuente única de información que complementa y contribuye a la teoría, investigación y la práctica» (Kazdin, 2001: 204). Mediante el análisis de los casos, y desde una perspectiva del psicoanálisis aplicado (Miller, 1998), se pretende abordar los modos de elaboración y significación del hecho victimizante de las personas que se han visto inmersas en el conflicto armado de las últimas décadas en el país. En concordancia, se seleccionaron tres casos de mujeres usuarias del proceso de acompañamiento terapéutico; teniendo en cuenta que a través de estas aproximaciones se logra develar qué enseñanzas se desprenden de estos casos respecto a lo que implica para un sujeto ser reconocido como víctima, su relación particular con el evento traumático y los alcances del proceso de intervención propuesto por el CAPsi.

\section{Participantes}

La ciudad de Cali es una de las ciudades de mayor recepción de víctimas del conflicto armado del suroccidente colombiano. ${ }^{2}$ En respuesta a esta problemática, el CAPsi ha trabajado en cooperación con organizaciones gubernamentales y no gubernamentales

2. En la revisión de fuentes acerca de las víctimas del conflicto armado en Cali no se encontró información consolidada. 
especializadas en la reparación integral a víctimas, ofreciendo un servicio de atención psicológica gratuita. Para llevar a cabo el propósito de esta investigación, se seleccionaron tres casos de mujeres usuarias de la línea de atención psicológica del CAPsi, cuyo motivo de consulta se relacionaba directamente con un hecho victimizante en el marco del conflicto armado. Otro criterio de selección es que las consultantes hubiesen culminado el proceso de atención, con el propósito de dar cuenta de los efectos del tratamiento psicoterapéutico. Para resguardar la identidad de las consultantes se utilizan pseudónimos en la presentación de resultados de investigación.

\section{Instrumentos}

Se trabajó en la recolección de datos a partir de la ficha socioeconómica, que se diligencia en la primera cita del proceso de atención psicológica; en esta las consultantes firmaron el consentimiento informado, según lo establecido por el Artículo 29 de la Ley 1090 de 2006. Posteriormente, se desarrolló la construcción de los relatos de los casos clínicos a partir de las categorías de análisis, con la información consignada en las historias clínicas del CAPsi, las cuales contenían el registro de cada sesión con sus respectivas notas de supervisión clínica.

\section{Procedimiento}

Esta investigación se desarrolló con posterioridad al proceso de atención psicológica de las mujeres víctimas en el CAPsi. Por tanto, el procedimiento constó de tres fases: primero, el acompañamiento terapéutico que tenía una duración de siete sesiones, y cada sesión fue supervisada por un profesional con experiencia en el campo de la psicología clínica. La segunda fase se trató del seguimiento y evaluación de los efectos del acompañamiento, a partir de la sistematización de las historias clínicas y una llamada de seguimiento que se efectuó a los tres meses de haber finalizado el proceso de intervención. La última fase fue la construcción de los relatos de los casos clínicos con la información de las historias clínicas y según las categorías de análisis. A continuación se presenta una gráfica de las fases de la investigación: 
GRÁFICA 1 Proceso de intervención-investigación en el CAPsi

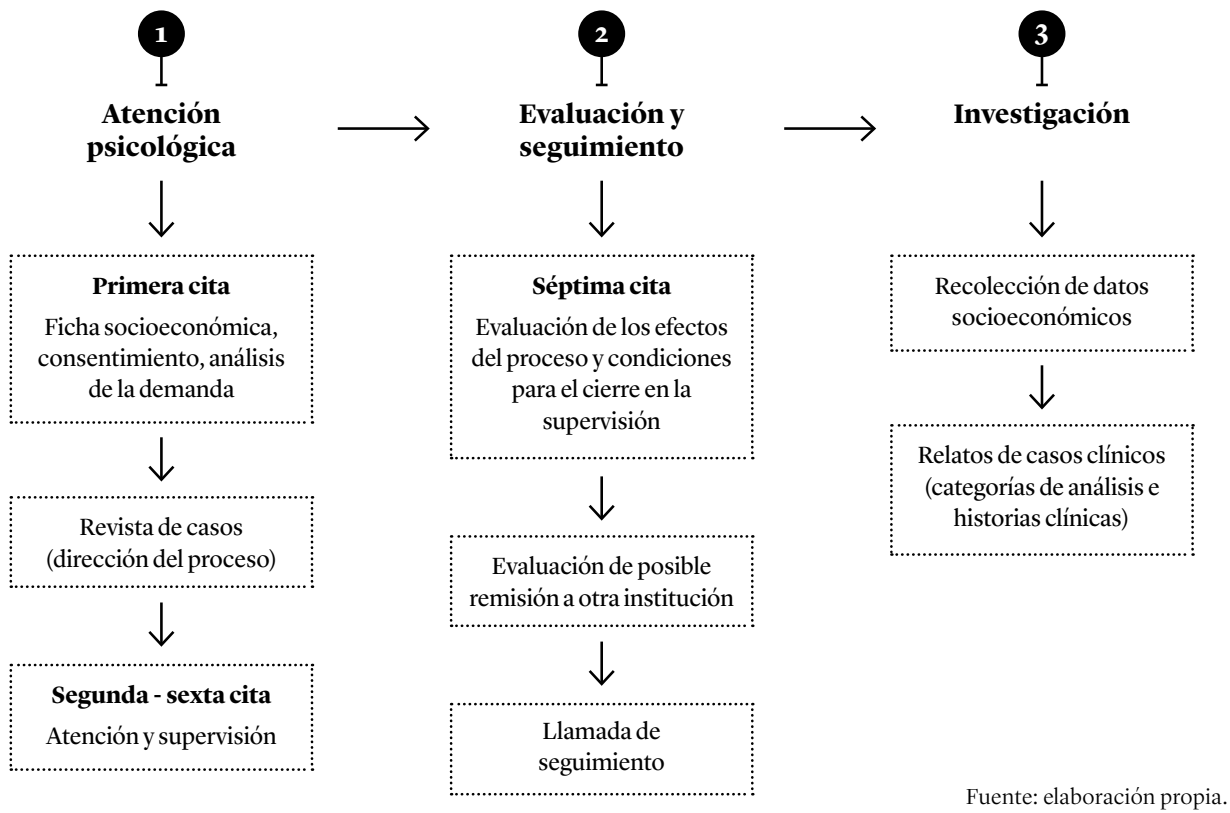

\section{Relato de los casos clínicos}

A continuación se presenta una narrativa descriptiva de los tres casos de víctimas del conflicto armado atendidos en el CAPsi. ${ }^{3}$

\section{Caso 1: Miralba}

Miralba es una mujer afrodescendiente de 44 años, proveniente de un municipio de la costa pacífica caucana, con educación primaria. Ella es remitida por la Unidad de Atención y Orientación a Víctimas (UAO) para que reciba atención psicológica. En el momento en que es atendida en el CAPsi, Miralba lleva dos meses viviendo en Cali en la casa de su hijastro; se desplazó desde un municipio del Pacífico del departamento del Cauca con sus dos hijas menores, quienes a raíz de lo sucedido están desvinculadas del sistema escolar, y con su madre, una mujer adulto mayor que tiene dificultades de movilidad.

3. Los casos que se presentan en este artículo fueron objeto de análisis en la tesis de maestría de Munévar (2017). 
Al momento de iniciar el proceso, la consultante presenta síntomas de angustia, como dificultades en el ciclo del sueño-en ocasiones solo duerme tres horas-; reporta que ha incrementado significativamente el consumo de cigarrillo-8 diarios-y refiere que tiene dificultades para manejar a sus hijas y no las ha inscrito al colegio; menciona además que en ese momento «no tiene ganas de luchar». Presenta pensamientos de minusvalía. Asimismo, ha tenido impases para conseguir la comida del día; muchas veces expone que sus hijas «la desesperan porque le piden muchas cosas».

Respecto a su historia de desplazamiento, Miralba narra que decidió irse de su pueblo porque, un día cualquiera de trabajo, unos hombres armados le quitaron la mercancía que debía vender en esa jornada y le dijeron que «no abriera la boca». La consultante explica que ahí llevaba viviendo dos años, y que primero había trabajado vendiendo pescado, pero en el último año laboraba vendiendo productos de limpieza, belleza y alimentos empacados a las personas que trabajaban en el río, en las minas o talando árboles.

Sobre su historia familiar, Miralba relata que tiene 5 hijos: las dos hijas con las que vive en Cali, una hija mayor que ya tiene familia y dos hijos de 20 y 15 años que hace dos años no viven con ella. Los cinco hijos los tuvo con su marido, con el cual dejó de vivir hace dos años, pues se dio cuenta de que la hija de una amiga era también hija de su esposo.

Además de esta situación, Miralba dice que durante muchos años su esposo la maltrató y que ella también le respondía las agresiones; narra, además, las discusiones con él de forma vívida pero poco coherente, lo que hace que resulte difícil seguir el hilo de sus ideas. Hace énfasis en que uno de los temas recurrentes en las discusiones de pareja era la «coquetería de él con las mujeres» y que él solía salir los fines de semana a beber licor sin regresar hasta el día lunes. La psicóloga trata de indagar sobre su infancia, pero presenta una narrativa incoherente, en la que se hacen notorios varios saltos en la temporalidad.

Durante el transcurso del proceso en el CAPsi-siete sesiones- la consultante relató otros dos hechos violentos que experimentó: uno de ellos en el pueblo en el que vivía con su madre y sus dos hijas, y el otro cuando vivía en la vereda con su esposo.

En un primer momento, relata las amenazas realizadas por los hombres armados, anteriormente descritas; en una sesión posterior, cuenta que unas semanas antes de ese suceso, mientras realizaba la venta de artículos en las afueras de su pueblo, vio el cadáver de una mujer por el cauce del río, comentando que, según algunas personas, había sido asesinada porque un grupo armado la había señalado de ser informante del Ejército (relata este hecho como una imagen fija y guarda silencio por un largo tiempo en la sesión). 
También narra que hace dos años, cuando vivía en otra vereda, quedó en medio de un enfrentamiento entre grupos armados y fue herida por una bala en el brazo. A pesar de lo fuerte de este evento, explica que le ha sido más difícil superar el último episodio, cuando fue amenazada por los hombres armados, aunque no se hayan dirigido a ella por nombre propio.

A raíz de estos eventos, la consultante presenta dificultades para dormir y constantes pesadillas. Cuenta que estas consisten en que «esos hombres me llevan»; respecto a este evento traumático, solamente una vez se pregunta si había exagerado en la decisión de dejar su casa, dado que en una ocasión logró comunicarse con una hermana que vive en su pueblo, quien le informó que había pasado por su casa y nadie preguntaba por ella. En otras ocasiones llega a expresar que piensa «muchas locuras», pero no explica en qué consisten; en el resto del proceso parece que las palabras de amenaza y la imagen de la mujer en el río se muestran como imposibles de relativizar; adicionalmente, la consultante prefiere no seguir abordando estas cuestiones, por lo que el CAPsi no insiste.

En paralelo al proceso que se desarrolla en el CAPsi, la UAO también remite a Miralba a psiquiatría y psicología en la EPS del régimen subsidiado. Es atendida en un centro de salud, donde inicia otro proceso con una psicóloga y un psiquiatra que le receta Sertralina ${ }^{4}$; este medicamento, según la paciente, le permite restablecer los ciclos de sueño. Además, el personal del centro de salud le ofrece empleo en varios oficios.

$\mathrm{Al}$ finalizar el proceso en el CAPsi, narra que le fue muy bien en el trabajo del puesto de salud; el señor que la contrató por una semana le dijo que apenas se presentara otro trabajo la llamaría. También narra que conoció, en el trabajo, a una mujer con la que se relacionó bien y la invitó a una iglesia cristiana. Esta mujer le comentó que cuando murió su esposo también le mandaron medicamento psiquiátrico pero ella no lo tomó, pues lo que le ayudó fue la Iglesia. La psicóloga del CAPsi señala a Miralba que ella ha mencionado el efecto benéfico que ha tenido el medicamento: y queda claro que eso no es todo, solo es una pequeña ayuda.

Habla de que la hija menor a veces suele exasperarla porque «me pide muchas cosas», pero esa semana pudo alimentar un poco mejor a sus hijas, gracias a que en la UOA la llamaron para entregarle unos bonos de alimentos y productos de aseo. Le informaron que el proceso para que la inscriban como beneficiaria del programa aún se demora, y no es del todo seguro que le den la ayuda económica.

4. La Sertralina es uno de los antidepresivos más usados para tratar cuadros de depresión, así como otros trastornos mentales. Pertenece al grupo de los inhibidores selectivos de la recaptación de serotonina (ISRS). 
La psicóloga del CAPsi le resalta la ayuda que le ha ofrecido la UOA, y le aconseja que también la utilice para vincular a sus hijas al sistema educativo, así sea que en un mes decida cambiarse de ciudad. La consultante refiere que en este momento sí quiere quedarse en la ciudad, dado que la psicóloga del puesto de salud le habló del caso de un joven que pasó por una situación similar y ahora ha logrado salir adelante; considera que en Cali hay más posibilidades para sus hijas. La psicóloga del CAPsi le recomienda no tomar una decisión precipitada al respecto.

Por último, la señora narra que una amiga del hijastro estuvo visitándola y le hizo unas trenzas con extensiones de cabello, Miralba cuenta que ya era hora de «consentirse un poco», porque se le estaba cayendo el cabello. Explica que ha podido sentirse mejor y «levantar cabeza», la profesional le dice que sí se ve mucho mejor y menos desanimada; se le pregunta qué cree que le ha ayudado a volver a «levantar cabeza», a lo cual responde que la trabajadora social y los medicamentos le sirvieron mucho para sentirse mejor, además de haber podido trabajar.

\section{Caso 2: Denisse}

Denisse es una mujer afrodescendiente de 38 años, originaria de la costa pacífica nariñense, lugar desde el cual tuvo que desplazarse a la ciudad de Cali, por haber sido víctima de conflicto armado. La consultante recibe un subsidio de la Unidad de Atención y Orientación a Víctimas. El proceso se desarrolló a lo largo de siete sesiones y fue remitida por el Consultorio Jurídico de la Universidad Icesi, debido a la afectación emocional manifestada.

Denisse decide iniciar el proceso de acompañamiento psicológico porque «lo ha perdido todo», narra: «no tengo fuerzas para yo seguir, no quiero vivir, estoy cansada, aburrida». Dice que ha sufrido mucho y que algunos días «quisiera que no amaneciera»; también manifiesta que ha tenido muchos problemas tanto con su hijo como con su esposo.

Respecto a la composición familiar, Denisse tiene tres hijos, de los cuales solo vive con el menor, de 16 años; el mayor consume sustancias psicoactivas y hace seis meses decidió devolverse a su pueblo de origen; el hijo del medio, de 19 años, se encuentra prestando servicio militar. Sus hijos son de su primer matrimonio, del cual su esposo falleció en un accidente en una mina. Hace 14 años ella está con su actual esposo y trabaja informalmente en «venta de minutos» en la calle.

Acerca de su historia de desplazamiento, expresa que en su pueblo tenía una tienda y un grupo armado le empezó a pedir «vacuna» (pagos de dinero para no hacerle daño) hasta que terminaron amenazando de muerte a su familia y abusando sexualmente de ella, y tuvo que dejar Nariño. En el transcurso del proceso, 
la consultante manifiesta que desde hace dos años no ha podido tener encuentros sexuales con su esposo, refiere que cuando estaba con él sentía que volvía a vivir esa situación horrible, y que no sabe por qué le pasa eso si no fue él el que le hizo eso; no le ha dicho a nadie lo que le sucedió, y dice: «me vergüenza decirle a mi esposo, me siento sucia». En las primeras sesiones el sufrimiento de Denisse era palpable y no sabía si quería continuar con su esposo, pues consideraba que él podía pensar que «le había sido infiel».

A lo largo del proceso y durante cada sesión surgía algo nuevo, hablaba de su historia de despojo, la cual inició a los 8 años cuando fue «robada» por una señora, que la llevó a la ciudad de Ipiales a laborar en oficios varios en casas de familia. Después, en su juventud, decidió volver a su pueblo natal, pero su madre ya había muerto y sus hermanos ya no vivían ahí; sin embargo, con el tiempo los pudo contactar. Al hablar de su tienda, que la obtuvo gracias a un préstamo con el Banco de la Mujer, explica que tuvo que dejarla por el conflicto armado, expresando lo siguiente: «todo lo que consigo tienen que quitármelo».

Durante las sesiones narró una serie de sucesos respecto a cómo se evidenciaba el conflicto armado en el territorio en el que vivía. Aunque ella no llegó a narrar el hecho del abuso sexual, sí cuenta que esos mismos hombres (abusadores) participaban en distintos crímenes en su pueblo: un vecino fue asesinado y otros tantos habían recibido amenazas. Ella describe cómo en las playas, al bajar la marea, aparecían cadáveres. En respuesta a esto Denisse decide escribir una carta al presidente de la republica y enviarla a Bogotá, para notificar la situación y solicitar auxilio, pero nunca recibió respuesta y se mostraba molesta y desilusionada por la impunidad en estos casos.

Denisse narra que en una ocasión anterior al abuso sexual, fue amenazada por aquellos hombres que le cobraban la «vacuna», y le apuntaron con un arma en la cabeza; dice que tenía tanto miedo que se orinó y se desmayó. Y al despertar, en el puesto de salud, su esposo le prometió tomar represalias contra esos hombres, pero ella le pidió que no lo hiciera; al final esto no sucedió y durante la sesión se le recalca que él la había escuchado.

La consultante habla sobre su historia de relaciones de pareja, expresa que el padre de sus hijos la maltrataba, y después de dos años de que falleciera, trabajando en una plantación, conoció a su actual esposo, el cual le ayudó a criar a sus hijos. Hace tres meses él se fue de la casa, pero la sigue visitando cada semana. Denisse refiere que muchas veces él le preguntaba qué le pasaba, que la notaba «distinta», ante lo cual ella guardaba silencio. Refiere que no está segura de querer continuar esa relación.

Denisse vivió varios momentos de despojo, pero a pesar de esto pudo construirse una vida con su esposo y fue una ventaja que él no se fuera del todo, pues la siguió 
visitando. Este elemento fue señalado por la psicóloga durante el proceso; también enfatizó la distinción entre los hombres que le han hecho daño (el difunto esposo y los hombres que abusaron de ella) y su esposo, mostrando cómo este no hace parte de la serie de agresores. Estas intervenciones partían de las palabras de la consultante, de sus propios dichos. Casi al final del proceso se da una breve ausencia del esposo de Denisse, con lo que ella pudo ubicar algo de su deseo al manifestar que «no quería que él se fuera», y decide buscarlo.

En los últimos encuentros narró que su esposo la había buscado para su cumpleaños y, en bajo tono de voz, decía que le había regalado lencería. Tuvieron un momento a solas, ella pudo decirle que necesitaba más tiempo y él aceptó, respetando su silencio. Ella se presentaba con ánimo para afrontar la vida, dejando atrás el afecto triste y sentimientos de inutilidad, afirmado que este espacio la había ayudado a sentirse mejor, a «no sentirse ahogada».

\section{Caso 3: Jenny}

Jenny tiene 12 años al momento de la consulta, es de origen mestizo, proviene de un municipio del norte del Valle del Cauca, vive con sus padres y su hermano mayor de 17 años, y cursa sexto grado en una institución educativa pública. La consultante es acompañada por su padre, quien manifiesta que ella ha tenido varias dificultades desde que presenció el fallecimiento de su hermana mayor, tres años antes, en el año 2013.

Relata que en este momento Jenny está mejor, dado que anteriormente presentó comportamientos muy preocupantes, como la tricofagias, bajo rendimiento académico y dificultades en la convivencia escolar. Actualmente, el señor refiere que a su hija, en ocasiones, «le dan cositas» que parecen interrumpir su proceso de aprendizaje en el colegio. Luego, matiza su temor frente a las dificultades de su hija diciendo que la considera «muy inteligente y capaz».

Cuando en el salón de clase hacen dictados, su hija escribe a lo largo de toda la hoja del cuaderno el nombre de su hermana fallecida. Este es uno de los comportamientos que más le preocupan; también han tenido que comprarle ropa nueva, dado que ha roto la ropa de la hermana fallecida, que ella usa, y su propia ropa. Por último, expone la tricofagia que su hija presentó el año pasado, motivo por el cual deciden buscar ayuda.

Después de escuchar el motivo de consulta del padre, se escucha a solas a Jenny, quien explica que hay una profesora en el colegio que la regaña con frecuencia y 
le asigna calificaciones más bajas que las de sus compañeras, incluso cuando ella ha sido quien les explica las tareas. La insatisfacción que esto le ha producido ha hecho que en varias ocasiones se retire a la cancha del colegio a llorar-al narrar esto su voz se corta-.

Para esclarecer esta problemática, la psicóloga indaga sobre las circunstancias actuales de la relación con la profesora, a lo cual la consultante manifiesta que hace un tiempo le celebraron el cumpleaños a la maestra con una fiesta sorpresa y quedó muy contenta; considera que desde entonces «no la ha vuelto a regañar tanto».

A lo largo del proceso, que tuvo una duración de cuatro sesiones, el discurso de Jenny es coherente y habla de diversos temas en relación con su interés por mejorar en el colegio, las relaciones de amistad que ha establecido y las inquietudes sobre su cuerpo, propias de la adolescencia. Al finalizar el proceso, afirma que en el colegio le ha ido muy bien, que no ha vuelto a llorar y que su madre solo ha tenido que asistir a reuniones de padres.

Al final, ella retoma la cuestión de su hermana y dice que la siente como «su ángel», afirma saber que no volverá a verla, pero que desde el cielo la cuidará; la psicóloga interviene resaltando que su hermana no está, pero ella puede seguir recordando los momentos que compartieron juntas.

En la última sesión, la madre solicita hablar con la psicóloga del proceso para saber de Jenny para saber cómo ha progresado su hija. La psicóloga le indica que tal vez hubo un momento en el que su hija atravesó por una crisis, tal y como lo señalaron en la primera cita, pero se ha evidenciado en las sesiones que Jenny ha podido superar ese suceso de horror. Se le explica a la madre que para ella es claro que su hermana ya no está, pero la seguirá recordando y queriendo.

La madre comparte una experiencia que vivió hace una semana, durante unas actividades grupales con víctimas en las que le proponían trabajar sobre los momentos en los que fueron «valientes». Su hija contó que sentía a su hermana como su ángel de la guarda, a pesar de que ya no estaba presente, y el grupo aplaudió a su hija. La psicóloga expone que Jenny ha podido dejar atrás el suceso referente al conflicto armado que vivió.

Al final, la madre comenta que en la EPS cambiaron nuevamente la psicóloga y les recomendó remitir a Jenny a psiquiatría. Sobre este punto, la profesional le sugiere no continuar con más procesos en salud mental y psiquiatría, explicándole que antes sí fueron necesarias esas intervenciones, pero actualmente no lo son, dados sus avances en la casa, el colegio y los espacios de psicología. 


\section{Discusión}

\section{Sobre la condición de víctima}

A través de las historias de estas tres mujeres puede observarse la gran vulnerabilidad que han experimentado desde su infancia, marcada por la repetición de hechos de exclusión social como el trabajo infantil, la violencia de género, habitar en territorios dominados por grupos armados y, en el caso de Denisse, el abuso sexual. En cada una de ellas se observa que a partir del hecho victimizante se presenta, en un primer momento, una serie de manifestaciones subjetivas de fragilidad, en las que se hace evidente el sufrimiento y los modos singulares de significación de cada hecho.

En los tres casos puede decirse que en un primer momento el único sostén es reconocerse como víctimas para poder acceder a la asistencia social, dada la fragmentación de los lazos sociales y la fragilidad subjetiva, consecuencia del evento de violencia que experimentaron. En esta línea puede pensarse que en un contexto como el colombiano, donde es poco lo que se trabaja en función de la inclusión social, reconocerse como víctimas resulta ser el único modo que encuentran las personas para restaurar su ciudadanía, a partir del cuerpo sufriente de la víctima (Arias, 2012).

En el caso 1 (Miralba), la consultante se encuentra en una condición de gran vulnerabilidad, por lo que se podría pensar que reconocerse como víctima le permitiría inscribirse en un discurso social y evocar acciones de solidaridad por parte de la sociedad (Gatti, 2016). En la primera sesión, Miralba se presenta como una víctima sufriente, en una posición muy frágil, refiriendo: «no tengo ganas de seguir luchando». Así, la posición desde la que se sitúa Miralba da cuenta de estar en la búsqueda de que el Otro le ayude, razón por la cual asume la remisión de la UAO al CAPsi. No obstante, en tal caso la cuestión se sitúa en determinar de qué manera ella puede hacer uso de ese reconocimiento, sin ubicarse en una posición de mero objeto de indemnizaciones por parte de las instituciones.

Con Denisse (caso 2), se hace evidente la existencia de una considerable brecha entre el centro y la periferia, en el hecho de recurrir a escribir una carta al presidente para denunciar a sus victimarios. Denisse no se remitió a ninguna autoridad local a la hora de denunciar los sucesos, sino que resolvió apelar al presidente, pero no recibió respuesta; manifiesta malestar ante la impunidad y por no recibir ningún tipo de apoyo institucional. La necesidad de apelar al presidente también da cuenta de lo que Pécaut (1999) llama desconfianza generalizada: una sensación de suspicacia hacia el contexto mismo provocada por una ausencia de referencias sociales que lo ayuden a «inscribir lo que le ocurre en una perspectiva de conjunto» (20), y que, 
a pesar de provenir de una experiencia de terror compartida por muchos, se vive como una experiencia individual.

Por otro lado, cuando el Estado se manifiesta en modos de intervención con las víctimas del conflicto armado, lo hace a través del sistema de salud, en el cual podríamos preguntarnos si la condición de víctima sufriente puede convertirse en una cronicidad o puede ser solo un momento de transición. Como ocurre en el tercer caso, Jenny se encuentra en medio de un sistema de salud desarticulado, entra en un circuito de patologización y se ve abocada, hasta cierto punto, a retornar una y otra vez al hecho victimizante. Al llegar al CAPsi, ella se ubica en un lugar de víctima, poniendo en acto la rotulación diagnóstica que ha recibido, tal como lo manifiesta su familia, el sistema de salud y la institución educativa. Por este motivo, en la primera sesión, ella narra acontecimientos en los que ha sido víctima, pero por parte de otros agentes, por ejemplo la profesora, a pesar de que actualmente ya no se encuentra en un momento crítico. Es probable que los problemas que se presentan en el colegio estén relacionados con el beneficio secundario que deriva de su condición de víctima. Tal es el caso de los momentos en los que ella se salía del salón de clases a llorar en el patio y cuando perdía varias materias. Estos actos eran leídos como efectos del «traumatismo» y la ubicaban en un lugar de víctima, sin ninguna responsabilidad subjetiva.

\section{Sufrimiento y trauma}

A partir del análisis de los tres casos resulta posible acercarse a la vivencia singular del trauma en estas mujeres y a las significaciones particulares de cada una de ellas, dejando como enseñanza que el trauma solo será definido a posteriori según las coordenadas dadas por el sujeto (Arciniegas, 2002). Respecto al trauma, en la narrativa que construye Miralba (caso 1), este se muestra como un acto anclado a un hecho anterior, y cobra un significado muy singular para ella. Primero unos hombres armados que dicen «no abra la boca», luego este acto es vinculado a una escena anterior: el cadáver de una mujer bajando por el río, que se presenta como una imagen fija en la cual, posiblemente, signa su muerte. Esa imagen se muestra como algo imposible de dialectizar. En este punto se muestra de un modo muy explícito cómo «lo indeleble de las imágenes traumáticas, se explican en la fisura operada por el sujeto, por un real ${ }^{6}$ no del todo simbolizado» (Gómez, 2004: 90). Esta imagen de la mujer en el

6. Lacan (2012) propuso la categoría de real para nombrar lo imposible de representar, aquello que «no cesa de no escribirse» en lo que decimos, recordamos, percibimos, etc. Por lo que no hace referencia a aquello que se percibe de la realidad física o la realidad social. Esta categoría surge a partir de lo que Freud (1920) designó como trauma, aquello que se repetía en sus casos, las escenas sexuales infantiles y 
río representa además la «compulsión de repetición» que habita el trauma (Freud, 2004), dado que la imagen se le presenta a Miralba una y otra vez evidenciando la persistencia del horror imposible de nombrar y de asimilar subjetivamente.

Para la consultante, el evento traumático aparece como un real que no está enlazado a nada, y como tal es un real que irrumpe en su más absoluta arbitrariedad (Hellemeyer, 2014). Este tiene efectos radicales en el lazo social de Miralba, dado que la irrupción del mismo la lleva a desvincularse de su sociedad y cultura para desplazarse a una ciudad como Cali, donde se manifiestan otras formas de segregación y de exclusión social.

Con Denisse (caso 2), al igual que con Miralba, la estructura del trauma se mantiene: el suceso desencadenante de la crisis -haber sido abusada sexualmente por unos hombres que pertenecían a un grupo armado- es asociado a un hecho anterior, la amenaza de muerte por esos mismos hombres apuntándole con un arma de fuego). Ante esto, su respuesta subjetiva fue la vergüenza y el silencio. Pasaron dos años en los que no habló con nadie de este suceso y solo fue posible hablar de ello en el CAPsi ante la garantía de confidencialidad. El silencio en este caso da cuenta de un imposible de decir frente a un acontecimiento traumático. Nótese cómo para Denisse no es posible contarle esto a su pareja, a pesar de que le permitiría explicarle sus dificultades en la intimidad; esta vergüenza se da en tanto sus victimarios la han reducido a objeto, sometiéndola a una sobreexposición tanto del cuerpo como del ser y dejándola sin dignidad (Figueroa, 2013: 290). El motivo de la vergüenza, según Figueroa (2013), es que el deseo ha sido aniquilado por la «introducción del objeto de goce encarnado», por lo cual se entiende que Denisse siente pudor por haberse convertido en objeto, carente de deseo, en lugar de un ser en falta pero «deseante» (289).

En el caso 3 puede observase la manifestación de un momento posterior al tiempo crítico del trauma: al inicio de su historial clínico Jenny presentó sintomatologías como la tricofagia, después de haber presenciado el asesinato de su hermana. Sin embargo, a lo largo del proceso de escucha del CAPsi, que va más allá de la condición de víctima, se logra que Jenny hable de otras experiencias propias de la adolescencia, como las relaciones de amistad y la relación con su cuerpo, cuestiones que desde la perspectiva del CAPsi no necesariamente deben ser objeto de un tratamiento.

En línea con lo anterior, durante el poco tiempo del proceso, se posibilita que la consultante hable de lo que ha podido elaborar del evento traumático: presenciar la

los sueños de los soldados que regresaban de la guerra. Al referirse a lo Real del trauma, Lacan propuso el neologismo troumatisme en francés (trou significa agujero) para dar cuenta del agujero que caracteriza lo traumático debido a la imposibilidad de simbolizarlo totalmente; estructuralmente no existe la posibilidad de decir todo sobre el trauma. 
muerte de su hermana. Ella da cuenta de la elaboración que ha construido a lo largo de estos años, en el sentido de que su hermana va a ser su «ángel de la guarda», aceptando que no va a volver. Jenny opta por la elaboración simbólica del hecho, ya que lo real del trauma no es del todo simbolizado (Hellemeyer, 2014). Esta rectificación subjetiva le permite a Jenny tomar distancia de la identificación imaginaria con su hermana y revincularse con su propio proyecto vital, integrando el recuerdo de su hermana a su propia historia.

\section{Efectos y límites del dispositivo CAPsi}

A lo largo del proceso de intervención realizada por el CAPsi en los casos de las tres mujeres, puede observarse que se logra apaciguar la angustia y mitigar la manifestación de los síntomas, posibilitando la construcción de una salida simbólica frente al trauma vivido, lo que implica poner en palabras algo de ese horror, y favorecer el restablecimiento de algunos de sus lazos sociales. Estos efectos se dan gracias al vínculo terapéutico que hace un llamado a la localización y rectificación subjetiva, y en el cual cada sujeto pueda buscar modos de elaboración ante su sufrimiento (Miller, 2004), siempre teniendo como ejes los principios éticos de la dignidad y la libertad humana.

Uno de los casos que presentaba mayores condiciones de vulnerabilidad por la fractura de sus lazos sociales, era Miralba (caso 1), pero a través de los diferentes entes interventores (Unidad de Orientación y Atención a Víctimas, Puesto de Salud y CAPsi), se logró cierta inclusión en la ciudad de Cali. Mediante el acompañamiento ofrecido en el CAPsi se contiene la angustia y la consultante recupera el ciclo normal del sueño, reduce voluntariamente el consumo de cigarrillo y recobra la capacidad de concentración en el trabajo cotidiano.

Un ejemplo del trabajo con otras instituciones es cuando Miralba inicia otro proceso psicológico en el centro de salud, durante el proceso en el CAPsi. En la intervención del centro de salud se le aconseja a Miralba quedarse viviendo en Cali, y en el CAPsi se decide no contradecir dicho consejo, asumiendo que el centro de salud constituye también un lugar de apoyo. En este sentido, el CAPsi opta por dejar abierta la pregunta sobre el lugar donde ella quisiera vivir, con el propósito de que ella se ubique como sujeto de decisión.

Según los informes del Centro de Memoria Histórica (2013), hay gran relevancia de los recursos socioculturales a través de los cuales las comunidades logran elaborar los daños causados por la violencia. Teniendo en cuenta esto, en el caso de Miralba, perteneciente a la comunidad afrodescendiente de la región del Pacífico colombiano, se evidencia el uso de estos recursos culturales y comunitarios como una vía para reconstituir los lazos sociales fracturados. 
En el trabajo de escucha del malestar de Denisse (caso 2) se logra apaciguar la angustia, lo cual se evidencia en las verbalizaciones de la paciente: «ya no me siento ahogada». A lo largo de la narrativa de Denisse puede pensarse que en el evento de abuso sexual ha vivido un encuentro con «un real» que le es imposible simbolizar (Gómez, 2004). Es posible pensar que para ella esto significó un punto final de su vida como mujer. Desde la perspectiva del CAPsi se buscó posibilitar una elaboración simbólica de ese imposible de decir, poner en palabras algo de ese horror que vivió sin caer en el imperativo de narrar el hecho traumático, sin forzarla a enfrentarse a sus recuerdos antes de que ella se sintiera preparada para hacerlo.

También puede evidenciarse que se logró delimitar el evento traumático, en palabras de Denisse: «no todo es malo». Asimismo, se posibilitó un reencuentro amoroso con el esposo, y enunciando una posición ante su deseo como mujer, ella logró llegar a afirmar: «no quiero que se vaya». Por otro lado, también se pudo vislumbrar un renovado interés por su proyecto de negocio propio, pensando nuevas formas de generar más ingresos para su familia.

El relato de esta mujer da cuenta del «íntimo vínculo entre trauma e impunidad. Es decir, la posibilidad de tramitación subjetiva de lo sucedido no es sin una operación en el campo de la ley» (Hellemeyer, 2014: 52). Es decir, que en el caso particular de Denisse, se percibe la necesidad de proponer un trabajo mediante una inscripción colectiva; por ello, al finalizar el proceso en el CAPsi, se le remitió a una organización de defensa de derechos de la mujer. Es probable que en los colectivos ella encuentre un modo de lazo social que le permita salir de un sufrimiento privado y silenciado, al ubicarse como un sujeto de derechos, para que su historia se inscriba en un discurso social, una inscripción política (Bravo, 2016). Infortunadamente, el seguimiento posterior al caso de la consultante no se efectuó, debido a la dificultad para contactarla y a la débil coordinación entre centros y dispositivos de apoyo a las víctimas.

Por último, en el tercer caso (Jenny) se evidencia un cambio de posición subjetiva, al dejar de ubicarse en el lugar de víctima pasiva. Esto se logró mediante la escucha atenta de las elaboraciones propias de la consultante y en la intervención con su madre, en la que se le recomendó suspender el circuito incesante de consultas médico-psiquiátricas y se le sensibilizó frente a la importancia de reconocer los avances de Jenny, más allá de su condición de víctima. Lo anterior muestra que, a pesar de que esta adolescente se ve determinada por un fenómeno social-el conflicto armado-, y un diagnóstico proveniente del discurso psiquiátrico-biológico, es ella misma, desde su condición de sujeto hablante, quien puede sobrepasar esas determinaciones, de modo que su «su autonomía y su creatividad serán siempre un 'más allá' de los límites que le imponen esos órdenes a los que esta sujetado» (Galende, 2008: 88). 


\section{Reflexión final}

A lo largo del presente texto se han mostrado -a través de la presentación de tres casos clínicos- diversos abordajes sobre el sufrimiento de las víctimas del conflicto armado colombiano y sus modos de tratamiento. Se ha realizado el recorrido desde posturas meramente medicalizantes, con un paradigma psiquiátrico-biologicista, y el reduccionismo jurídico de la víctima, hasta posturas que proponen abordajes como la reivindicación de los derechos vulnerados y las salidas simbólicas al sufrimiento. Esto se ha puesto en contraste con la propuesta de intervención clínico-comunitaria del CAPsi, mostrando así la pertinencia de un espacio de escucha para las personas víctimas del conflicto armado.

$\mathrm{El}$ anterior recorrido es importante porque existen posturas teóricas que consideran que la atención a víctimas a partir de la escucha del caso por caso solo remite a la perpetuación de la condición de víctima como sujeto pasivo y subalterno, exponiendo su cuerpo sufriente. Bajo estos supuestos, para la víctima solo queda la querella, la palabra parlanchina o el silencio traumático (Gatti, 2016). Pero nos podemos preguntar: ¿solo es posible la querella o el silencio traumático? Quizás sea posible propender por un espacio en el que se ofrezcan otras posibilidades, como la rectificación subjetiva, la cual implica un posicionamiento del sujeto en el que el lazo social no se signe solamente por el sufrimiento o la violencia.

La experiencia de atención psicosocial a víctimas del conflicto armado en el CAPsi permite valorar la importancia de una escucha que propicie la elaboración simbólica del horror vivido (Figueroa, 2013; Hellemeyer, 2014). Los casos de estas mujeres nos enseñan que este modo de nombrar o significar será singular y remitirá a sus historias siempre enmarcadas en su contexto sociocultural. Por tal motivo, se propone que las intervenciones psicosociales sigan las coordenadas dadas por el saber de cada sujeto, uno por uno, tomando distancia de los procesos protocolizados y masificados que suelen perpetuar el lugar de la víctima como cuerpo sufriente y como objeto pasivo de intervenciones. También es necesario que se creen redes entre instituciones asistenciales con el fin de complementar las atenciones psicoterapéuticas a nivel individual y familiar con asesorías en temas de derechos, grupos de apoyo e instancias colectivas de reivindicación política. 


\section{Referencias}

Aguilera, A. (2013). Componentes epistemológicos y metodológicos de la atención psicosocial a víctimas de guerra y violencia política en Colombia. Tesis Psicológica, 8(1), 68-79.

Arciniegas, L. (2012). Sujeto, trauma y síntoma. En: Restrepo-Espinosa, M. H. Salud mentaly desplazamiento forzado (pp. 39-49). Bogotá: Universidad del Rosario.

Arias-Marín, A. (2012). Teoría crítica y de derechos humanos: hacia un concepto crítico de víctima. Nómadas, Revista Crítica de Ciencias Socialesy Jurídicas, 36, 1-30. Recuperado de: http:// revistas. ucm.es/index.php/NOMA/article/view/42298/40258

Barrios, M. (2014). Cuando se ama para eludir la culpa. En: Sawiche, P. y Stillo, B. Relaciones violentas: entre el amory la tragedia (pp. 47-58). Buenos Aires: Grama.

Bolaños, T. (2011). Víctimas y el conflicto armado. Sobre la aplicación de la Ley de Víctimas en Colombia. Revista de Derechos Fundamentales, 6, 51-70.

Botero, C. (2004). Efectividad de una intervención cognitivo-conductual para el trastorno por estrés porstraumático en excombatientes colombianos. Universitas Psychologica, 4(2), 205-219.

Bravo, O. (2016). Patologías de la guerra: trauma, testimonio y olvido. Madrid: Grupo 5.

Castro, X. (2016). Salud mental y atención psicosocial. Reflexiones a partir de la experiencia de un dispositivo de escucha y su impacto en la rehabilitación de la cronicidad mental. En: Bravo, O. (ed.). Pensar la salud mental: aspectos clínicos, epistemológicos, culturales y políticos (pp. 121156). Cali: Universidad Icesi. Recuperado de: http://repository.icesi.edu.co/biblioteca_digital/ bitstream/10906/81081/1/bravo_pensar_salud_2016.pdf

Castro, X. y Munévar, M. (2016). Consultorio de Atención Psicosocial: clínica y lazo social. En: Colegio Colombiano de Psicólogos. Experiencias significativas en salud mentaly psicología. Bogotá: Colegio Colombiano de Psicólogos. Recuperado de: http://colpsic.org.co/aym_image/ files/EXPERIENCIAS_SIGNIFICATIVAS.pdf

Centro de Memoria Histórica (2013). Los daños y los impactos sobre las víctimas. En: ¡Basta ya! Colombia: Memoria de guerra y dignidad. Bogotá: Pro-Offset.

Desviat, M. (2015). De la reforma psiquiátrica a la salud mental colectiva. Madrid: Grupo 5.

Efraime, B. (2017). Postconflicto y salud mental. Cali: Universidad Icesi.

Figueroa, M. (2013). La vergüenza en las víctimas del conflicto. Desde el Jardín de Freud, 13, 275-291.

Freud, S. (2004). Más allá del principio de placer. En: Obras completas, vol. XVIII (pp. 1-62). Buenos Aires: Amorrortu.

Galende, E. (2008). Psicofármacos y salud mental: la ilusión de no ser. Buenos Aires: Lugar. 
García, Y. (2012). Las víctimas del conflicto armado en Colombia frente a la ley de víctimas y otros escenarios de construcción de memorias: una mirada desde Foucault. Justicia Juris, 18(2), 459-485.

Gatti, G. (2016). El misterioso encanto de las víctimas. Revista de Estudios Sociales, 56, 117-120.

Giraldo, M. (2016). Del hecho traumático a la decisión de la víctima. IX Jornadas de la NEL: Violencias y pasiones. Sus tratamientos en la experiencia analítica. Guayaquil. Recuperado de: https://antenafapolchile.com/2016/o6/o6/del-hecho-traumatico-a-la-decision-de-la-victima-por-maria-cristina-giraldo/

Gómez, G. (2004). Traumatismos de guerra: memoria y olvido. Desde el Jardín de Freud, 4, 84-101.

Grupo de Memoria Histórica (2010). Bojayá: la guerra sin límites. Bogotá: Taurus.

Gustin, T. y Palau, C. (2013). Aplicación de la Ley 1448 de 2011 por parte de la gobernación del Valle del Cauca en el plan de acción territorial de víctimas del conflicto armado interno (Tesis de pregrado). Cali:Universidad Icesi. Recuperado de: http://bibliotecadigital.icesi.edu.co/biblioteca_digital/ bitstream/10906/76502/1/aplicacion_ley_gobernancion.pdf

Gutiérrez-Peláez, M. (2017). Retos para las intervenciones psicológicas y psicosociales en Colombia en el marco de la implementación de los Acuerdos de Paz entre el Gobierno y las FARC-EP. Avances en Psicología Latinoamericana, 35(1), 1-8.

Hellemeyer, A. (2014). El testimonio y el pudor. En: Destinos del testimonio: víctima, autor, silencio. Buenos Aires: Letra Viva.

Jimeno, M. (2008). Lenguaje, subjetividad y experiencias de violencia. En: Ortega, F. (ed.). Veena Das: Sujetos de dolor, agentes de dignidad (pp. 261-291). Bogotá: Colección Lecturas CES.

Kazdin, A. (2001). Métodos de investigación en psicología clínica. México D. F.: Pearson.

Kleinman, A., Das, V. y Lock, M. (1997). Introduction. En: Social Suffering (pp. ix-xxv). Berkeley: University of California.

Kovalskys, J. (2006). Trauma social, modernidad e identidades sustraídas: nuevas formas de acción social. Psykhe, 15(2), 13-24.

Lacan, J. (2012). Radiofonía. En: Lacan, J. Otros escritos (pp. 433-468). Buenos Aires: Paidós.

Latorre, E. (2010). Memoria y resiliencia. Estudio de la memoria de las víctimas del conflicto armado en el departamento del Magdalena: Presentificación, visibilización, catársis y resiliencia. Prolegómenos, Derechos y Valores, 25, 95-109.

Londoño, N., Muñiz, O., Patiño, C., Jaramillo, G., Raigoza, J., Restrepo, D. y Rojas, C. (2005). Salud mental en víctimas de la violencia armada en Bojayá. Revista Colombiana de Psiquiatría, 34(4), 493-505.

Miller, J. A. (1998). Introducción al método psicoanalítico. Barcelona: Paidós. 
Miller, J. A. (2004). La utilidad social de la escucha. Virtualia, 10, 2-4. Recuperado de: http:// www. revistavirtualia.com/storage/articulos/pdf/cquMKv49dZ8pQdVyCp1gfzS3wAozGkQoHFO2paSt.pdf

Ministerio de Salud y Protección Social (14 de marzo de 2017). El Programa de Atención Psicosocial y Salud Integral a Víctimas - PAPSIVI. Recuperado de: https://www.minsalud.gov.co/proteccionsocial/promocion-social/Victimas/Paginas/papsivi.aspx

Munévar, M. (2017). Tras las pistas de una invención: Sistematización de una experiencia de atención psicológica breve a víctimas del conflicto armado (Tesis de maestría). Universidad Icesi, Cali.

Pécaut, D. (1999). Configuración del espacio, el tiempo y la subjetividad en un espacio de terror: el caso colombiano. Revista Colombiana de Antropología, 35, 8-35.

Quintero, D., Recalde, G., Gómez, D. y Flechas, D. (2015). Informe complementario en el marco del Auto 333 de 2015 en el proceso de seguimiento a la Sentencia T-025 de 2004. Cali: Universidad Icesi.

Rebolledo, I. y Rondón, L. (2010). Reflexiones y aproximaciones al trabajo psicosocial con víctimas individuales y colectivas en el marco del proceso de reparación. Estudios Sociales, 36, 40-50. Recuperado de: http://res.uniandes.edu.co/view.php/648/index.php?id=648

República de Colombia (2011). Ley 1448 de Víctimas y restitución de tierras. Recuperado de: http:// wsp.presidencia.gov.co/Normativa/Leyes/Documents/ley144810062011.pdf

Salgado, D., Nobles, D. y Ruiz, V. (2011). Indemnización del dolor y objetivación del trauma en víctimas del conflicto armado. Revista de la Facultad de Psicología, Universidad Cooperativa de Colombia, 7(12), 127-133.

Wilches, I. (2010). Lo que hemos aprendido sobre la atención a mujeres víctimas de violencia sexual en el conflicto armado colombiano. Revista de Ciencias Sociales. Recuperado de: http:// res.uniandes.edu.co/view.php/652/index.php?id=652 